Relations industrielles

Industrial Relations

\title{
Participation aux bénéfices
}

\section{Ernest Moreau}

Volume 7, numéro 1-2, décembre 1951, mars 1952

URI : https://id.erudit.org/iderudit/1023078ar

DOI : https://doi.org/10.7202/1023078ar

Aller au sommaire du numéro

Éditeur(s)

Département des relations industrielles de l’Université Laval

ISSN

0034-379X (imprimé)

1703-8138 (numérique)

Découvrir la revue

Citer cet article

Moreau, E. (1951). Participation aux bénéfices. Relations industrielles / Industrial Relations, 7(1-2), 51-53. https://doi.org/10.7202/1023078ar
Résumé de l'article

Ce bref compte-rendu du dernier congrès des Chambres de Commerce de la province de Québec apporte des suggestions nouvelles pour l'étude et l'application de cette idée en marche: la participation aux bénéfices.
Tous droits réservés (C) Département des relations industrielles de l’Université Laval, 1952
Ce document est protégé par la loi sur le droit d'auteur. L’utilisation des services d'Érudit (y compris la reproduction) est assujettie à sa politique d'utilisation que vous pouvez consulter en ligne.

https://apropos.erudit.org/fr/usagers/politique-dutilisation/ 


\section{Participation aux bénéfices}

\section{Ernest Moreau}

Ce bref compte-rendu du dernier congrès des Chambres de Commerce de la province de Québec apporte des suggestions nouvelles pour l'étude et l'application de cette idée en marche: la participation aux bénéfices.

Les dirigeants des nombreuses Chambres de Commerce de la province de Québec viennent d'étudier, à l'occasion de leur récent congrès, le problème de la participation aux bénéfices.

C'est un événement qui mérite d'être mentionné et qui prouve que l'idée est en marche et que les éléments qui ont la mission de l'appliquer s'en préoccupent sérieusement.

Les industriels et les commerçants, soucieux de leurs responsabilités sociales, avaient déjà effleuré ce sujet l'année précédente en semblable circonstance; aussi ont-ils voulu en faire le thème principal de leurs études cette année.

Sans vouloir reprendre l'exposé de ce thème, connu de tous, il est opportun de dire que les employeurs s'accordent pour admettre qu'il est urgent « d'introduire des éléments du contrat de société dans le contrat de travail de façon à améliorer la condition générale du travailleur ». Et que les directives contenues dans la lettre pastorale collective des Evêques de la province de Québec sur le « problème ouvrier en regard de la doctrine sociale de l'Eglise », au sujet de la participation aux bénéfices, ont été le motif principal de l'étude en congrès de cette question.

Monsieur J.-A. Archambault, le nouveau président de la Chambre de Commerce de la Province, en faisant.l'exposé du thème, déclarait que «l'employeur devait prendre dans l'ordre social, les mêmes responsabilités qu'il doit prendre dans le domaine économique et que s'il ne manifeste pas cet esprit de risque et d'aventure dans le domaine de la sociologie, il sera dépasşé par l'Etat et par les masses. » Il ajoutait cependant que la participation aux bénéfices est un

MOREAU, ERnest, président de la Chambre de Commerce de Québec. 
sujet qui a passionné, divisé et même amené de sérieuses discussions, et, que pour l'instant, il était urgent de rallier les opinions et faire appel aux initiatives, tout en agissant avec prudence, car les temps modernes n'autorisent pas l'insécurité.

Le problème de la participation aux bénéfices n'est pas nouveau, car, sous des formes variées, il a trouvé son application dans les milieux industriels de bien des pays. Il a même subi la forme de l'obligation imposée dans beaucoup de pays actuellement sous le contrôle du communisme et aussi dans la plupart des pays de l'Amérique du Sud. Mais, dans notre pays de même qu'aux EtatsUnis, il a réalisé des hausses et des baisses.

Actuellement, il est propagé d'une façon méthodique par un organisme connu sous l'appellation de "Council of Profit Sharing Industries 》 fondé en 1947 et qui groupe principalement les entreprises américaines et aussi un certain nombre de maisons canadiennes. Cette association accepte dans ses rangs « un employeur qui, en vertu de n'importe quel arrangement, paie à tous ses employés, en plus de salaires réguliers normaux, des montants spéciaux comptan's ou différés, et établis non seulement sur les qualifications personnelles ou de groupe, mais sur la prospérité de l'entreprise comme un tout ».

Il faut admettre cependant qu'ils sont rares les hommes d'affaires de la province de Québec qui ont adhéré à cette association, car dans ce domaine comme dans beaucoup d'autres ils sont réfractaires à tout organisme où l'élément anglais prédomine, non par antipathie, mais par prudence à cause des différences de conceptions au point de vue de l'application des idées économicosociales.

Sommes-nous en retard? ou sommes-nous trop prudents ? D'après les statistiques fournies au cours de la discussion du problème, il est reconnu que, proportion gardée, les expériences dans nos milieux sont assez nombreuses et il ne faut pas s'attendre à une application générale dans ces années difficiles où les profits sont incertains.

Avant d'appliquer la méthode de la participation aux bénéfices, il faut être certain de la stabilité des profits, car rétrograder ou discontinuer ce procédé de distribution aux employés, c'est travailler dans le sens contraire à nos objectifs.

Les congressistes ont été suffisamment informés au sujet du mode d'application ainsi que pour la mise en oeuvre de ce plan.

Comme il était bien entendu que cette étude devait être faite d'une façon objective, le congrès s'est terminé sans l'approbation d'aucun voeu ou de réso- 
lution favorables ou défavorables, laissant aux intéressés le soin d'utiliser à bon escient la volumineuse documentation qui leur a été fournie.

Cependant il me semblerait intéressant de faire connaître le contenu d'un projet suggéré par monsieur François LePelch, ingénieur et consultant industriel dont l'expérience dans ce domaine mérite d'être mentionnée. Il suggère l'idée suivante:

«Tout en refusant pour le moment de prendre parti pour ou contre le système, il ne peut rester sans écho dans le domaine de l'action constructive.

En face de l'action collective ouvrière, à tendance revendicative, les chefs d'entreprise ont le devoir impérieux et urgent de mettre en action un programme constructif tendant à promouvoir les bases d'une nouvelle coopération solide et durable, dans l'intérêt commun.

Si la participation aux profits se révélait, lors d'une expérience valable, comme un des moyens efficaces, il faudrait alors en recommander et en organiser l'emploi.

A cet effet, il est suggéré:

1. - La nomination d'un comité assurant la liaison entre les exécutifs des associations professionnelles de la Province. Il serait chargé de trouver dans quelques activités-clés de la Province, un nombre très réduit d'affaires moyennes, dont la direction soit favorable à l'essai du plan.

L'affaire-pilote devrait avoir une organisation interne se rapprochant des conditions techniques et psychologiques nécessaires à la mise en route normale du plan.

2. - La préparation finale technique et psychologique de l'affaire, le choix du plan, l'aide à la mise en opération seraient assurés par des experts qualifiés, travaillant dans la langue d'usage de l'entreprise, et dont l'esprit de vente de leurs services n'aurait pas détruit la notion de service public. Ces experts bénéficieraient de l'appui technique total de l'expérience considérable des membres du Profit Sharing Council.

3. - L'expérience tentée étant utile à la communauté, le comité spécial étudierait les modalités de l'appui matériel et moral.

4. - L'expérience devrait durer un délai raisonnable de deux années, au bout desquelles un congrès spécial serait réuni et étudierait les résultats présentés par les experts, avec les commentaires des intéressés eux-mêmes, tant patrons que délógués ouvriers.

5. - Le rôle délicat et important de toutes les Chambres de Commerce serait de protéger ces expériences loyales et de dénoncer publiquement les tentatives de sabotage qui seront nombreuses, variées, et venant de tous les horizons, même les plus inattendus. »

Nous recommandons particulièrement cette initiative aux administrateurs de la Chambre de la Province et, en attendant, nous félicitons ceux-ci d'avoir placé devant l'opinion publique ce problème de la participation aux bénéfices.

Déjà la presse a commenté favorablement cette idée en marche. 